\title{
THE EFFECT OF SILVER VERSUS TITANIUM DIOXIDE NANOPARTICLES ON POLY METHYLMETHACRYLATE DENTURE BASE MATERIAL
}

\author{
Yasmine Galaleldin Thabet *, Dina Mohamed Moustafa** and Sara Fikry El-Shafei****
}

\begin{abstract}
Materials and methods: Three groups of heat cured acrylic resin specimens were prepared; the first was Group C made of conventional heat cured acrylic resin, the second group was Group $\mathrm{Ag}$ where the acrylic resin specimens were incorporated with 5\% Silver nanoparticles, and the third group Ti had its specimens incorporated with 5\% Titanium oxide nanoparticles. The specimens were tested for microbial colonization using direct culture and serial dilution methods. The specimens were then further tested for surface roughness.
\end{abstract}

Results: There were higher bacterial counts in specimens of group C (control) $51.12 \pm 4.7198$ than those with $\mathrm{Ag}$ nanoparticles $32.44 \pm 2.7701$ and $\mathrm{TiO} 2$ nanoparticles $31.92 \pm 2.2159$. The difference was statistically significant between the control group (C) and the Ag nanoparticles group and between the control group and the $\mathrm{TiO} 2$ nanoparticles group. However, there was a statistically non-significant difference between the Ag nanoparticles group and $\mathrm{TiO} 2$ nanoparticles group. Statistical analysis by ANOVA for repeated measures showed statistically non-significant difference between the three groups regarding the surface roughness.

Conclusions: $\mathrm{Ag}$ and $\mathrm{TiO} 2$ nanoparticles had a similar antimicrobial effect when added to PMMA, The addition of 5\% of $\mathrm{Ag}$ and $\mathrm{TiO} 2$ nanoparticles has not affected the surface roughness of PMMA.

KEYWORDS: Titanium, Silver, Nanoparticles

* Associate Professor, Oral and Maxillofacial Prosthodontics department, Faculty of Dentistry, Ain Shams University, Cairo, Egypt.

** Assistant Lecturer of Microbiology and Immunology department, Faculty of Dentistry, The British University in Egypt.

*** Lecturer, Oral and Maxillofacial Prosthodontics department, Faculty of Dentistry, The British University in Egypt, Cairo, Egypt. 


\section{INTRODUCTION}

Up to date, there is no material that can fulfill the role of polymethyl methacrylate (PMMA) particularly in the field of removable prosthodontics. PMMA is the mostly used denture base material as it is light in weight, insoluble in the oral fluids and easily repaired. It also has a low cost and superior esthetics. However, it has several limitations as it undergoes polymerization shrinkage and has inferior mechanical properties. Recently, several nanosized fillers have been added to improve the properties of PMMA. ${ }^{(1)}$ Nanoparticles have a very high surface area and they affect the mobility of the polymer chains at their interfaces. Thus, the addition of a small quantity of nanoparticles drastically transforms the properties of a polymer. ${ }^{(2,3)}$

Silver (Ag) nanoparticles are smaller than other fillers and possess distinct physical, chemical, and biological properties. ${ }^{(4)}$ They have been used to enhance the mechanical and thermal properties of denture base materials. They also possess an antimicrobial activity as they prevent the adherence of both bacteria and fungi on the surfaces of denture bases. ${ }^{(5)}$ It has been recommended to incorporate lower concentrations of $\mathrm{Ag}$ nanoparticles into denture base materials as they impart a black color to the denture bases when used in higher concentrations. ${ }^{(6)}$ The use of PMMA incorporated with Ag nanoparticles promotes better oral health in patients with removable prosthesis thus improves their quality of life. ${ }^{(7)}$

Titanium dioxide (TiO2) nanoparticles are also used for reinforcement of denture base materials. They are biocompatible, non-toxic, and chemically stable. They also have high strength, resist corrosion, and have an antimicrobial activity. They alter the electrical, optical, chemical, and physical properties of polymers. They also cause drastic improvement of the mechanical properties of denture base materials when compared to unmodified acrylic resins. ${ }^{(8-10)}$ It was reported that the enhancement of the properties of the polymers was only achieved with lower concentrations of $\mathrm{TiO} 2$ and that higher concentrations had an adverse effect on the final hybrid polymer. ${ }^{(8)}$

The texture of the surface of a denture base of a removable prosthesis should be smooth with minimal surface roughness as it directly contacts the oral mucosa. A rough surface is unhygienic and tends to retain plaque and microorganisms which affect the oral health. It was reported that the recommended threshold to reduce the plaque accumulation is $0.2 \mu \mathrm{m}$ and that accumulation of microorganisms increases significantly when the surface roughness is more than $2 \mu \mathrm{m}$. ${ }^{(11)}$

The favorable mechanical properties achieved with the addition of different nanofillers renders them ideal for improvement of the qualities of denture base materials. However, the effect of these fillers on the surface properties of the denture bases as well as their antimicrobial activity should be in consideration. Hence, this study was conducted to compare the effect of adding $\mathrm{Ag}$ and $\mathrm{TiO} 2$ nanoparticles on the antimicrobial effect and surface roughness of PMMA denture base material. The first null hypothesis was that no difference would be found in the antimicrobial action of the denture base material by addition of $\mathrm{Ag}$ and $\mathrm{TiO} 2$ nanoparticles. The second null hypothesis was that no difference would be found in the surface roughness of the denture base material by addition of $\mathrm{Ag}$ and $\mathrm{TiO} 2$ nanoparticles.

\section{MATERIALS AND METHODS}

The denture base material used in this study was heat cured poly methyl methacrylate (Acrostone, Acrostone Dental Factory, El Salam City, Egypt). Two types of nanoparticles were used in this study, Silver (Ag) and Titanium Dioxide (TiO2). They were prepared According to the manufacturer's instructions. 


\section{Silver Nanoparticles Preparation}

Silver (Ag) nanoparticles were prepared using chemical reduction method. Microwave irradiation of silver nitrate $(\mathrm{AgNO} 3)$ solution in ethanolic medium using polyvinylpyrrolidone (PVP) as a stabilizing agent was carried out. Ethanol is used because it acts as a reducing agent in the presence of microwaves. The solvent was then evaporated at mild temperature to obtain the Silver in powdered form. ${ }^{(12)}$

\section{Titanium Dioxide Nanoparticles preparation}

Anatase particles were prepared by precipitation from homogenous solution using titanium (IV) isopropoxide as precursor in aqueous solution acidified with nitric acid to $\mathrm{PH} 2$ using a water to titanium mole ratio of about 200. ${ }^{(13)}$

\section{Nanocomposite mixture Preparation}

Three groups of specimens were constructed, each group consisting of $n 25=$ samples.

Group C was considered the Control group and the specimens of this group were constructed out of conventional Heat Cured PMMA (Acrostone, Acrostone Dental Factory, El Salam City, Egypt) without the addition of Nanoparticles.

Group Ag had its PMMA powder incorporated with $5 \%$ by weight silver nanoparticles prior to monomer addition and specimen construction. Silver particle average size was less than $45 \mathrm{~nm}$ and their shape was spherical.

Group Ti had its PMMA powder incorporated with $5 \%$ by weight titanium nanoparticles also prior to monomer addition and specimen construction. Titanium particle average size was less than $15 \mathrm{~nm}$ and their shape was spherical.

Both nanocomposite mixture groups were prepared by gradual addition of the nanoparticles to the PMMA powder under continuous stirring until a homogenous mixture is obtained.

\section{Specimen construction}

Base plate wax (Cavex Set up Wax; Cavex, Haarlem, the Netherlands) was cut into $15 \times 2 \pm 0.01$ $\mathrm{mm}$. These discs were then flasked in dental stone (Fujirock EP; GC, Leuven, Belgium). Separating medium was applied on the stone surface, then the upper half of the flask was seated and filled with another layer of stone. After setting, the flasks were opened, and the wax discs were removed, thus leaving the molds empty for the denture base material.

All three previously prepared PMMA nanocomposite powder mixtures had their monomer added to them according to the manufacturer's instructions. Once the mixtures reached the dough stage, they were packed into the molds and pressed using a hydraulic press. The flasks were then placed in a curing unit and cured at $74^{\circ} \mathrm{C}$ for $90 \mathrm{~min}$ and then to $100^{\circ} \mathrm{C}$ for $30 \mathrm{~min}$. The flask was removed and left to cool gradually, and then opened and the acrylic resin specimens were finished and polished using a tungsten carbide bur. (Fig. 1)

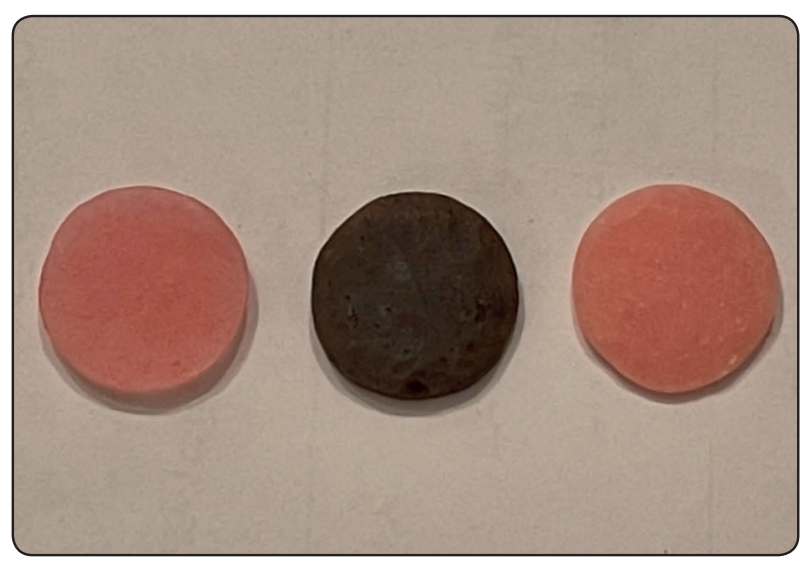

Fig. (1) Specimens from the three groups: Control (C), Silver (Ag) and Titanium (Ti). 


\section{Investigations}

\section{Microbial investigation}

For the C. albicans adherence assay: Disinfection of the specimens with $70 \%$ alcohol was carried out and then ultrasonic cleaning with sterile distilled water. These were then soaked in artificial saliva containing $2 \times 10^{\wedge} 6$ cells of C. albicans (ATCC $10231)$ at $37^{\circ} \mathrm{C}$ for 48 hours. The specimens were then placed in sterile tubes containing $1 \mathrm{ml}$ of Sabouraud dextrose broth for 24 hours and vortexed for 10 minutes. (Fig.2)

The tubes were centrifuged for 5 minutes at 4500 rpm followed by the extraction of the acrylic resin specimens and clustered pellet. The C. albicans in the detached cell suspension was counted by direct culture tests and serial dilutions to determine colony forming units (CFUs) as follows:

\section{A) Direct culture test:}

One hundred microliters $(100 \mu \mathrm{l})$ of the centrifuged pellet were taken and cultured in plates filled with SD agar and incubated for 24 hours at $37 \circ \mathrm{C}$. After incubation, colonies of C. albicans were counted in the plates using a marker pen colony counter. Colonies covering the whole surface of the plate were considered overgrowth.

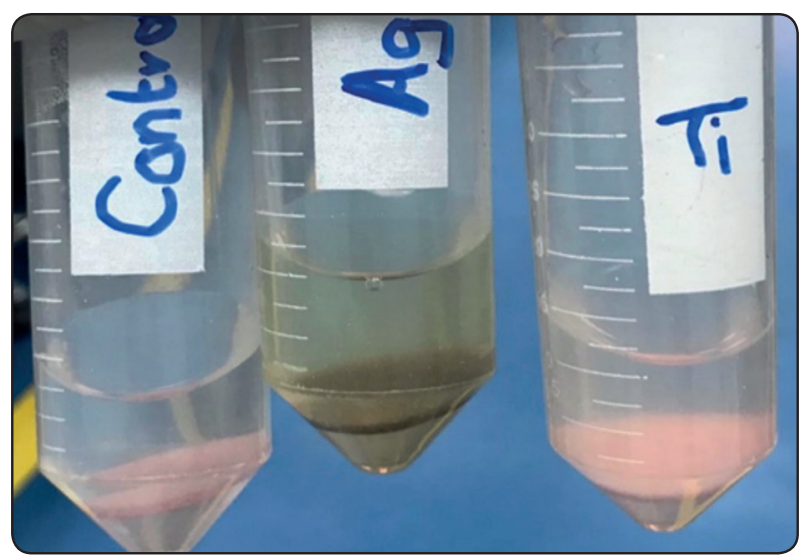

Fig. (2) Acrylic resin specimens of Control, Silver and Titanium groups in falcon tubes containing artificial saliva.

\section{B) Serial dilution method:}

Five tubes were filled with $900 \mu \mathrm{l}$ of normal saline. One hundred microliters of the concentrated pellet were added to the first tube (1/10) and mixed. A total of $100 \mu \mathrm{l}$ was aspirated from the first tube and added to the second tube $\left(1 / 10^{\wedge} 2\right)$. The second tube was then mixed, and $100 \mu \mathrm{l}$ were aspirated from it, before being added to the third tube $\left(1 / 10^{\wedge} 3\right)$. The solution in the third tube was mixed, followed by aspirating $100 \mu \mathrm{l}$ and adding it to the fourth tube $\left(1 / 10^{\wedge} 4\right)$, which were mixed as well. 100 $\mu \mathrm{l}$ were added to the fifth tube $\left(1 / 10^{\wedge} 5\right)$ from the fourth tube and mixed. Finally, $100 \mu \mathrm{l}$ were aspirated from the fifth tube and, after proper mixing, discarded. At this stage, all tubes contained $900 \mu \mathrm{l}$ of diluted concentration of the broth. SD agar plates were cultured with $10 \mu \mathrm{l}$ of diluted concentration of each tube. The plates were incubated for 24 hours at $37^{\circ} \mathrm{C}$. Colonies were counted as previously mentioned in the direct culture test. (Fig. 3)

\section{Surface roughness}

The surface roughness (Ra value) of 25 specimens from each group was measured using Perthometer (Mahr, Germany). Each sample was placed on the provided platform so that the Perthometer probe is in slight contact with its surface. The probe

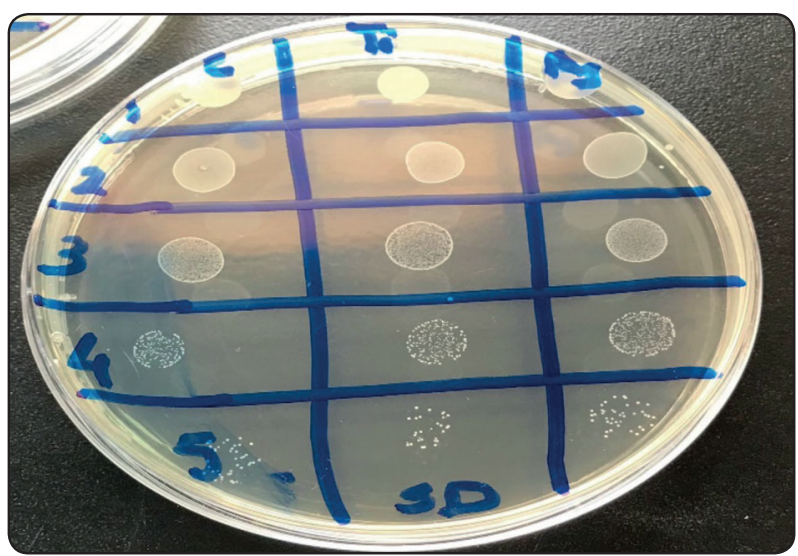

Fig. (3) Plate count of different dilutions for the Control (C), Silver (Ag) and Titanium (Ti) 
was passed over the entire specimen surface, and 3 readings were recorded per specimen. An average of these readings was taken as the value of surface roughness for every specimen.

$\mathrm{G}$ power software for windows version 3.1.9.4 was used to calculate the sample size. The results were collected and statistically analysed. The collected data were tested for normality by checking distribution of data and calculating the mean values and normality curves. Numerical Data were presented by mean, and standard deviation (SD). ANOVA for repeated measures was used for comparison between the groups. Tukey's HSD was used as a Post Hoc test. The significance level was set at $\mathrm{P} \leq 0.05$.

\section{RESULTS}

Table 1 shows that there were higher bacterial counts in group C (control) 51.12 \pm 4.7198 than in the other two groups with $\mathrm{Ag}$ nanoparticles $32.44 \pm 2.7701$ and $\mathrm{TiO} 2$ nanoparticles 31.92 \pm 2.2159 . Statistical analysis by ANOVA for repeated measures showed statistically significant difference between the three groups. Tukey's HSD was used as a Post Hoc test which showed there was a statistically significant difference between the control group and the Ag nanoparticles group and between the control group and the $\mathrm{TiO} 2$ nanoparticles group. However, there was a statistically non-significant difference between the Ag nanoparticles group and $\mathrm{TiO} 2$ nanoparticles group.

The results for the comparison of the surface roughness in the three groups were presented in Table 2. Although the specimens of the group with Ag nanoparticles show increased surface roughness (Ra values) $1.1719 \pm 0.2049$ than both the group with $\mathrm{TiO} 2$ nanoparticles $1.1683 \pm 0.1983$ and group C (control) 1.121 \pm 0.1759 . Statistical analysis by ANOVA for repeated measures showed statistically non-significant difference between the three groups.
TABLE (1) Shows the mean, standard deviation, and $P$ value of ANOVA for repeated measures test for comparison of the number of bacteria in the three groups.

\begin{tabular}{|c|c|c|c|}
\hline Groups & Mean & $\begin{array}{c}\text { Std. } \\
\text { Deviation }\end{array}$ & Pvalue \\
\hline $\begin{array}{l}\text { Group C } \\
\text { (Control) }\end{array}$ & $51.12^{\mathrm{a}}$ & 4.7198 & \multirow{3}{*}{$<.00001$} \\
\hline Group Ag & $32.44^{b}$ & 2.7701 & \\
\hline Group Ti & $31.92^{b}$ & 2.2159 & \\
\hline
\end{tabular}

Similar letters indicate non-significance.

TABLE (2) Shows the mean, standard deviation, and $P$ value of ANOVA for repeated measures test for comparison of the surface roughness in the three groups.

\begin{tabular}{|c|c|c|c|}
\hline Groups & $\begin{array}{c}\text { Mean } \\
(\mu \mathrm{m})\end{array}$ & $\begin{array}{c}\text { Std. } \\
\text { Deviation }\end{array}$ & Pvalue \\
\hline $\begin{array}{l}\text { Group C } \\
\text { (Control) }\end{array}$ & 1.121 & 0.1759 & \multirow{3}{*}{.584924} \\
\hline Group Ag & 1.1719 & 0.2049 & \\
\hline Group $\mathrm{Ti}$ & 1.1683 & 0.1983 & \\
\hline
\end{tabular}

\section{DISCUSSION}

The addition of nanoparticles to PMMA denture bases has proved to have many beneficial effects. ${ }^{(14)}$ Among the most widely used materials in this field are Silver and Titanium oxide nanoparticles. To the best of our knowledge, no previous studies have compared the effect of these two materials when incorporated into PMMA powder.

For standardization purposes, $5 \%$ by weight of both Silver and Titanium Oxide nanoparticles was added to the study groups. Silver nanoparticles were proven to have an antimicrobial effect when used at concentrations of $5 \%$ by weight and were therefore used in this concentration. ${ }^{(15)}$ For the Ti group, $5 \%$ by weight $\mathrm{TiO} 2$ nanoparticles were added to the PMMA powder. Studies have mentioned that this is the highest concentration that can be incorporated 
into PMMA without causing toxicity, although it might affect other mechanical properties. ${ }^{(8,16)}$

The first null hypothesis was rejected as the results of this study showed significantly reduced numbers of bacterial colonies in the PMMA specimens incorporated with $\mathrm{Ag}$ and $\mathrm{TiO} 2$ nanoparticles in comparison to the control group. These results coincide with the results of several previous studies. ${ }^{(15-17)}$

A study reported that $\mathrm{Ag}$ nanoparticles inhibited the formation of Candida albicans nanofilm on denture bases and had an antifungal activity. The incorporation of 5\% $\mathrm{Ag}$ nanoparticles inhibited the adhesion of Candida albicans on the surface of the denture bases. ${ }^{(15)}$ Another study proposed the use of Ag nanoparticles with nystatin or chlorhexidine digluconate for treatment of denture stomatitis. ${ }^{(16)}$ Furthermore, a study confirmed that Ag nanoparticles inhibit bacterial growth on the surface of PMMA and release Ag ions that induce an antibacterial action against Escherichia coli and S. epidermidis. ${ }^{(17)}$

It has been reported that the antimicrobial activity of $\mathrm{Ag}$ nanoparticles is due to the formation of reactive oxygen species (ROS) or due to the release of free silver ions from the denture bases. The mode of action depends on the method of synthesis, $\mathrm{Ag}$ nanoparticle dispersion and $\mathrm{Ag}$ concentration in the PMMA. ${ }^{(17)}$

Similarly, TiO2 nanoparticles have an antimicrobial effect specially on candida species. In fact, it has been found that the antimicrobial activity is not only limited to various bacterial strains but also on fungi and viruses. ${ }^{(18)}$ The antimicrobial activity of $\mathrm{TiO} 2$ nanoparticles is due to its surface properties as well as the photocatalytic production of cytotoxic oxygen radicals. ${ }^{(19)}$ Studies have shown that the antimicrobial effect of $\mathrm{TiO} 2$ nanoparticles was intensified when exposed to UV-A light. ${ }^{(20)}$ The results of this study showed that there was no significant difference in the antimicrobial activity of the $\mathrm{Ag}$ and $\mathrm{TiO} 2$ nanoparticles.

The effect of the added nanoparticles on the surface roughness depends on the quantity of the filler as well as the degree of homogeneity within resin matrix. The interface between the nanoparticles and the PMMA matrix, as well as the agglomeration of the nanoparticles may also influence the surface roughness of the hybrid PMMA. (7) Although the Ag nanoparticles slightly increased the surface roughness of PMMA specimens more than the $\mathrm{TiO} 2$ nanoparticles. Yet, the difference between the surface roughness of the specimens of the three groups was statistically nonsignificant. Thus, the second null hypothesis was accepted.

These results are similar to a previous study where there was a statistically nonsignificant difference between the denture base specimens without nanoparticles and those with silver nanoparticles with different concentrations. ${ }^{(15)}$ However, they are different from those of other studies where there was a significant increase in the surface roughness of specimens of PMMA incorporated with $\mathrm{TiO} 2$ nanoparticles when compared to pure PMMA. ${ }^{(21,22)}$ The dissimilarity of the results may be due to the reduced size of the $\mathrm{TiO} 2$ nanoparticles used in this study (less than $15 \mathrm{~nm}$ ). According to a previous study, the size of the filler particles affects the surface characteristics of resin composites where the Ra value increases as the size of the filler particles increased. ${ }^{(23)}$

According to the results of this study, there is no difference regarding the antimicrobial effect and the surface roughness of PMMA with the addition of $5 \% \mathrm{Ag}$ and $\mathrm{TiO} 2$ nanoparticles. Further studies are recommended to evaluate and compare the effect of other concentrations on different properties of PMMA.

\section{CONCLUSIONS}

- $\quad \mathrm{Ag}$ and TiO2 nanoparticles had a similar antimicrobial effect when added to PMMA.

- The addition of 5\% of $\mathrm{Ag}$ and $\mathrm{TiO} 2$ nanoparticles has not affected the surface roughness of PMMA.

\section{ACKNOWLEDGMENTS}

We thank Dr. Marwa Sabet (Professor at Ain Shams University) for her comments on the manuscript. 


\section{REFERENCES}

1. Sheng, T. J., Shafee, M. F., Ariffin, Z., Jaafar, M. Review on poly-methyl methacrylate as denture base materials. Malays. J. Microsc. 2018; 14:1-16

2. Ahmed MA, El-Shennawy M, Althomali YM, Omar AA. Effect of Titanium Dioxide Nano Particles Incorporation on Mechanical and Physical Properties on Two Different Types of Acrylic Resin Denture Base. World J Nano Sci Eng. 2016;06(03):111-9

3. Marquis D.M., Guillaume E., Chivas-Joly C. Properties of Nanofillers in Polymer. Nanocomposites and Polymers with Analytical Methods, John Cuppoletti, IntechOpen. 2011

4. Corrêa JM, Mori M, Sanches HL, da Cruz AD, Poiate E Jr, Poiate IA. Silver nanoparticles in dental biomaterials. Int J Biomater. 2015;1-9.

5. Monteiro, D.R.; Takamiya, A.S.; Feresin, L.P.; Gorup, L.F.; de Camargo, E.R.; Delbem, A.C.; Henriques, M.; Barbosa, D.B. Susceptibility of Candida albicans and Candida glabrata biofilms to silver nanoparticles in intermediate and mature development phases. J. Prosthodont. Res. $2015,59,42-48$.

6. Alla R.K., Swamy K.N., Vyas R., Konakanchi A., Guduri V., Gadde P. Influence of Silver Nanoparticles Incorporation on Flexural Strength of Heat-cure Acrylic Denture Base Resin Materials. Annu. Res. Rev. Biol.2017; 17(4): 1-8

7. Carlos F.A., Rene G.C., Germán V.S., Susana A.T. Antimicrobial Poly (methyl methacrylate) with Silver Nanoparticles for Dentistry: A Systematic Review. Appl. Sci. 2020; 10,4007

8. Gad M.M., Abualsaud R. Behavior of PMMA Denture Base Materials Containing Titanium Dioxide Nanoparticles: A Literature Review. Int. J. Biomater. 2019

9. Chatterjee A. Properties improvement of PMMA using nano TiO2. J.Appl. Polym. Sci., 2010; 118 (5):2890-2897.

10. Paul L., Ravichandran R., Kumar K.H., Nair V.V., Janardanan K., Deepthi V.S. Effect of titanium dioxide nanoparticles incorporation on tensile and impact strength in two different acrylic denture base resins. Int. Dent. J. Stud. Res. 2020;8(2):65-74.

11. Quirynen M., Marechal M., Busscher H.J., Weerkamp A.H., Darius P.L., van Steenberghe D. The influence of surface free energy and surface roughness on early plaque formation. An in vivo study in man. J Clin Periodontol, 1990; 7: 138-144

12. Pal A, Shah S, Devi S. Mater. Chem. Phys. 2009; 114: 530532.
13. Vorkapic, D.; Matsoukas, T. J. Colloid Interface Sci. 1999; 214: 283-291.

14. Akay C., Avukat E.N. Effect Of Nanoparticle Addition On Polymethylmethacrylate Resins. Acta sci. dent. sci. 2019;3(7): 91-97.

15. Li Z, Sun J, Lan J, Qi Q. Effect of a denture base acrylic resin containing silver nanoparticles on Candida albicans adhesion and biofilm formation. Gerodontology.2016;33(2):209-16.

16. Monteiro DR, Silva S, Negri M, Gorup LF, de Camargo ER, Oliveira R, Barbosa DB, Henriques M. Antifungal activity of silver nanoparticles in combination with nystatin and chlorhexidine digluconate against Candida Albicans and Candida glabrata biofilms. Mycoses. 2013;56(6):672-680

17. Lyutakov, O.; Goncharova, I.; Rimpelova, S.; Kolarova, K.; Svanda, J.; Svorcik, V. Silver release and antimicrobial properties of PMMA films doped with silver ions, nanoparticles and complexes. Mater. Sci. Eng. C. 2015, 49, 534-540.

18. De Dicastillo C.L., Correa M.G., Martínez F.B., Streitt C., Galotto M.J. Antimicrobial Effect of Titanium Dioxide Nanoparticles. In Antimicrobial Resistance-A One Health Perspective; IntechOpen: London, UK, 2020.

19. Li Q., Mahendra S., Lyon D.Y., Lyon D.Y., Brunet L., Liga M.V., Li D., Pedro J J Alvarez P.J.J. Antimicrobial nanomaterials for water disinfection and microbial control: potential applications and implications, Water Res. 2008;42(18):4591-602.

20. de Dicastillo C.L, Patiño C, Galotto M.J., Vásquez-Martínez Y., Torrent C., Alburquenque D., et al. Novel hollow titanium dioxide nanospheres with antimicrobial activity against resistant bacteria. Beilstein J. Nanotechnol. 2019; 10:1716-1725.

21. Chowdhury A.R., Kaurani P., Padiyar U.N., Meena S., Sharma H., Gupta A. Effect of Addition of Titanium Oxide and Zirconium Oxide Nanoparticles on the Surface Roughness of Heat Cured Denture Base Resins: An InVitro study. SVOA Materials Science \&Technology, 2021, 3(3) Pages: 36-44.

22. Alwan S., Alameer S. The effect of the addition of silanized Nano titania fillers on some physical and mechanical properties of heat cured acrylic denture base materials. JBCD 2021;27(1):86-1.

23. Marghalani H.Y. Effect of filler particles on surface roughness of experimental composite series. J Appl Oral Sci. 2010;18(1):59-67 